ARTICULOS ORIGINALES Rev Chil Salud Pública 2013; Vol 17 (2): 124-131

\title{
Evaluación económica del programa de fluoración del agua de beber en Chile
}

\section{RESUMEN}

Objetivo: Elpropósito de este estudio fue realizar un Análisis Costo-Efectividad (ACE), desde el punto de vista social, de añadir fluoruros al agua de beber para prevenir caries dentales en niños de 12 años de edad, versus el status-quo, en la realidad chilena.

Método: En un ACE, los costos de programas alternativos se miden y los resultados se valoran en unidades de efectividad. Para el caso, dientes con historia de caries dental, usando el indice de dientes cariados, obturados o perdidos debido a caries dental (COPD). La comparación se bizo respecto de un grupo no expuesto a fluoruración del agua. Todos los costos y los beneficios se calcularon desde una perspectiva de análisis económico social. Los datos sobre efectividad se obtuvieron de la literatura.

Los costos de tratamientos dentales se calcularon usando aranceles de referencia. Costos en gastos a nivel familiar en términos de pérdida de producción, viajes hacial desde el dentista se tomaron en cuenta. Los costos y beneficios se descontaron usando una tasa de descuento del 3\%.

Resultados: El costo de la intervención, sobre la estrategia de no intervención se comparó con los beneficios obtenidos por la intervención. El cociente costo-efectividad indica que, en promedio, este programa dio lugar a aborros en Pesos Chilenos (2009) $(\mathrm{RCH} \$)$ de 8.930,49 por diente afectado por caries evitado. Esta mejora se alcanzó a un costo anual de $\mathrm{RCH} \$ 841,33$ por niño.

Conclusiones: Considerando nuestros supuestos y para situaciones equivalentes a las que prevalecen en Chile, un programa de fuoruración del agua representa un uso eficiente de los recursos de la comunidad.

Palabras claves: Evaluación económica, Análisis Costo-Efectividad, caries dental, fuoruración, Chile.

\section{Economic evaluation of the Fluoridation Program of DRINKING WATER IN CHILE}

\footnotetext{
ABSTRACT

Objective: This paper models the cost-effectiveness, from a social viewpoint, of a dental caries prevention program for children 12 years of age using water fluoridation, compared with nonintervention (or status quo).

Method: In cost-effectiveness analysis (CEA,) the costs of alternative programs are measured as economic costs and the results are valued in units of effectiveness. In this case, teeth
} 
with history of dental caries were measured using the DMFT index. The control group was a group that was not exposed to water fluoridation. Data on effectiveness were obtained from the literature.

Costs of dental treatments were calculated using current established prices. Family expenses for the treatment were based on lost income, as well as transport costs to and from the clinic. The costs and benefits were calculated using a discount rate of $3 \%$.

Results: To find the cost of the intervention, the non-intervention strategy was compared to the additional benefits gained as a result of the intervention. The cost-effectiveness ratio indicates that on average, this program saved \$8,930.49 Chilean pesos at the 2009 value $(\mathrm{RCH} \$)$ per cavity-affected tooth. This was achieved with an annual cost of $\mathrm{RCH} \$ 841.33$ per child.

Conclusions: Taking into account our assumptions, for situations similar to those in Chile, a water fluoridation program would be an efficient use of community resources

Key words: Economic evaluation, Cost-effectiveness analysis, dental caries, fluoridation, Chile.

\section{INTRODUCCIÓN}

Por más de 60 años investigaciones de laboratorio, clínicas y comunitarias han estudiado el uso de fluoruros en la prevención de la caries dental. Su uso es aceptado como un mecanismo seguro, efectivo, eficiente y apropiado para la prevención de la caries dental ${ }^{1,2}$. Aún más, su uso en la prevención de la caries dental se reconoce además como uno de los diez grandes logros de la salud pública del siglo pasado ${ }^{3}$. El uso del agua de beber como un vehículo para fluoruros $(\mathrm{F})$ es reconocido internacionalmente, junto con la leche y la sal, como, un vehículo altamente costo-efectivo ${ }^{1}$, apoyando su implementación como una medida de salud comunitaria.

En Chile, como en muchos otros países, la fluoración del agua potable es una de las principales estrategias para la prevención y control de la caries dental ${ }^{4,5}$. Intervención que comenzó en 1953 y dio como resultado una sustancial disminución de la caries dental en las ciudades donde fue implementada ${ }^{6}$. El programa nacional fue interrumpido en $1975 \mathrm{y}$, a mediados de los ochenta, la prevalencia de caries aumentó a niveles críticos, equivalente a países carentes de programas de fluoración 7 . En julio de 1981 se lanza un nuevo esfuerzo legislativo con la creación del "Programa Nacional de Fluoración de los Abastos de Agua Potable" (Decreto Supremo No. 915 con fecha 8/07/1981).

En 1985, el primer programa de fluoración de agua bajo esta nueva legislación comenzó en la Región de Valparaíso. Desde entonces, el programa se ha expandido y en la actualidad aproximadamente $82,3 \%$ de la población chilena se abastece por sistemas de agua cuya concentración de fluoruro ha sido ajustada a niveles óptimos para la prevención de caries dental ${ }^{8}$.

Una evaluación económica (EE) se define como "el análisis comparativo de líneas alternativas, en términos de sus costes y consecuencias para ayudar a las decisiones de política"9. La EE es parte integral del proceso de planificación y de entrega de servicios de salud. La EE es además un componente de toda evaluación objetiva de cualquier programa preventivo ${ }^{10}$. Existe un volumen considerable de literatura acerca del valor económico de la fluoración del agua de beber y sus resultados continúan apoyando su uso $^{11,12}$. Sin embargo, en América Latina, con la excepción de evaluaciones económicas de programas de fluoración de la sal ${ }^{13-15}$, en leche fluorurada ${ }^{16}$, y en fluoruros tópicos ${ }^{17}$, no existen evaluaciones económicas publicadas de programas de prevención de caries.

Las EE son contexto específicas, por tanto, no pueden ser usadas para informar políticas en otras poblaciones ${ }^{18}$. Por consiguiente, una evaluación económica de programas en Chile permitiría conclusiones que reflejen los usos y prácticas para esta realidad nacional, y no la de países donde las EE de programas de fluoruración del agua están disponibles. El propósito de este estudio es estimar la costo-efectividad, desde el punto de vista social (societal), de añadir fluoruros al agua 
de beber para prevenir caries dentales en niños de 12 años de edad, versus una comunidad urbana equivalente sin exposición a un programa de fluoruracion del agua de beber (statu-quo), bajo la realidad chilena.

\section{MATERIAL Y MÉTODOS}

La forma de evaluación económica que se usó en este estudio fue el análisis costo-efectividad (ACE). En un ACE los costos de programas alternativos son medidos como costos económicos y los resultados son valorados como unidades de efectividad'. Esto es, dientes con historia de caries dental, medidos usando el índice de dientes cariados, obturados perdidos debido a caries dental (COPD). Para estimar el costo-efectividad incremental, el modelo asume que 80.000 niños* de 12 años de edad estaban bajo el programa de fluoración del agua de beber. El comparador de esta intervención fue una comunidad urbana equivalente no expuesta un programa de fluoruración del agua de beber (o statu-quo).

\section{Costos}

Desde una perspectiva social, se identificaron e incluyerontodos los costosimportantes y relevantes de la intervención, costos de coordinación del programa, costos de los controles de calidad, etc. Los costos fueron identificados en unidades físicas apropiadas, esto es, número de exámenes de orina realizados, etc. Luego se les otorgó valor usando los costos reales del mercado.

Los gastos totales en el grupo bajo el programa incluyeron los costos del tratamiento dental de los niños de este grupo más los costos asociados con la operación del programa de fluoruración del agua. Éstos fueron: el sueldo del coordinador del programa; los costos de insumos, el alquiler de oficina, el mobiliario de oficina y los costos generales, como electricidad, alquiler, etc. Estos costos fueron estimados en base al costo habitual para el alquiler de oficina y electricidad, el teléfono, limpieza y otros servicios basado en aquellos para Santiago de Chile. Los costos compartidos y los costos del personal se repartieron de acuerdo al tiempo que dedicaron a la intervención. El espacio para la oficina y el uso de vehículos se establecieron en base al uso real por parte el programa.

Los costos de los insumos del programa se calcularon en base al cargo autorizado por la incorporación la fluoruración del agua potable, por parte el Ministerio de Economía de Chile a las empresas de agua potable. Estos valores se revisan cada cinco años y varían en gran escala en las diferentes comunas de Chile ${ }^{19}$. Sin embargo, la directriz indica que el costo debe reflejar su valor verdadero. Para los cálculos se tomó como referencia 1,84 pesos por metro cúbico de agua, que corresponde al costo de una comuna de ingresos medios de la ciudad de Santiago ${ }^{20}$. Se estimó que el consumo de agua para un niño de 6 a 12 años era igual en cada año y correspondía, en promedio, a 196 litros al día ${ }^{19}$.

Para el cálculo de los recursos familiares utilizados para tratamientos dentales (es decir, transporte y pérdidas de productividad), asumimos que la duración de una visita dental era 1,5 horas y que un viaje era necesario para cada tratamiento. Este tiempo incluyó el tiempo de transporte público desde y hacia el consultorio de salud y para el tratamiento dental. Las pérdidas de productividad de trabajo se calcularon para el adulto que acompaña al niño multiplicando el número de visitas dentales requeridas para cada grupo por el sueldo mínimo por hora en 2009. Los costos por pérdida de productividad para niños no se calcularon. Los gastos de transporte se calcularon para el adulto de que acompañaba multiplicando el número de viajes por el número de visitas dentales requeridas para cada grupo.

Los costos del tratamiento dental se calcularon en base a los aranceles obturaciones y extracciones del sector público. Se asumió que el costo por tratamiento representaba el costo total de la prestación.

Además, el Ministerio de salud tiene pautas de vigilancia epidemiológica para programas de fluoruración comunitaria. Estos protocolos determinan que se debe realizar un examen dental una vez cada cinco años y que se debe monitorear

\footnotetext{
* 80.000 niños sería la población aproximada de niños de 12 años de edad en la ciudad de Santiago, Chile.
} 
la excreción de urinaria ${ }^{21}$. Las pautas nacionales establecen que se deben tomar 20 muestras de orina cada 6 meses $^{22}$.

Los costos totales para el grupo de comparación fueron aquellos asociados con el tratamiento dental. Cuando fue necesario, para ajustar por inflación, se uso el índice de precio al consumidor (IPC) de los años 2003 al 2009. Todos los gastos y los ahorros se calcularon en pesos chilenos al valor del año 2009 (RCH\$) (1 US\$ = RCH\$ 600,00).

\section{Efectividad}

Como medida de efectividad se utilizó el índice COPD. Este índice es la suma de los dientes cariados $(\mathrm{C})$, obturados $(\mathrm{O})$ y perdidos $(\mathrm{P})$ por caries en la dentición permanente. Los resultados obtenidos cuando aún no se introducía el programa de agua de beber en la ciudad de Santiago, indican que los niños de 12 años de edad tenían un COPD de 3,13 ${ }^{23}$. Por otra parte, datos de 2007 indican que, niños de 12 años residentes en Santiago, que tuvieron acceso a agua fluorurada desde su nacimiento, mostraron una experiencia de caries dental en sus dientes permanentes (COPD) de $1,87^{24}$. Esto que representa una reducción en los índices de caries dental del 40,3\%. La diferencia de 1,26 COPD entre las dos medias es estadísticamente significativa ( $p<0,01)$ (Ver Tabla 1).

El análisis primario del ACE se efectuó bajo los supuestos siguientes:

a) Que el aumento en el componente cariados (C) y perdidos (P) del índice COPD ocurre con la misma tasa en cada año del estudio.

b) Que los costos asociados al tratamiento de los dientes con caries y las extracciones ocurren en el mismo año del incremento.

c) Los niños en la comunidad bajo el programa y en el grupo de comparación tiene el mismo incremento anual de caries.

d) Todos los de dientes con caries reciben restauraciones de una superficie. Esto es porque en el sector público los aranceles son iguales, independientes del número de superficies dentales afectadas.

e) Todos los dientes afectados con caries serían tratadas.

f) Que el coordinador del programa de fluoruración trabaja en el programa con una carga equivalente al 0,10 de un horario a tiempo completo.

g) Se supuso que cada niño recibe un examen bucodental cada 5 años.

h) Los costos de cada uno de los 6 años se descuentan al valor presente usando una tasa de descuento del $3 \%{ }^{25}$. Los resultados clínicos no se descontaron.

i) Que todos los beneficios del programa se acumulan sólo hasta la edad 12 años de edad.

j) Que una sesión es necesaria para la obturación de cada diente cariado.

k) Que no hay necesidad de reemplazar restauraciones de dientes permanentes.

\section{Análisis de sensibilidad}

Como es posible que algunos de los supuestos utilizados en el análisis primario tengan un grado de incertidumbre, se realizó un análisis de sensibilidad de una y dos vías para poner a prueba la robustez de los resultados con los valores de parámetro claves'. Los parámetros que se modificaron fueron: el tipo de descuento, usando los valores de

Tabla 1. Promedio y desviaciones estándares de dientes cariados, obturados perdidos (COPD) en niños de 12 años residentes en Santiago antes y después de exposición a agua fluorurada y disminución en el índice COPD

\begin{tabular}{|l|c|c|c|}
\hline & Status-quo\# & $\begin{array}{c}\text { Con exposición agua } \\
\text { fluorurada }\end{array}$ & Disminución \\
\hline Dientes cariados & 1,82 & $1,05(1,68)$ & 0,77 \\
\hline Dientes perdidos & 0,26 & $0,07(0,36)$ & 0,19 \\
\hline Dientes obturados & 1,05 & $0,75(1,33)$ & 0,30 \\
\hline $\begin{array}{l}\text { Dientes obturados cariados } \\
\text { perdidos (COPD) }\end{array}$ & $3,13(2,73)$ & $1,87(2,05)$ & 1,26 \\
\hline
\end{tabular}

\# Sin exposición agua fluorurada 
0\% y del 6\%; y el rango de variabilidad de eficacia, mejores y peores, en reducción de caries por el agua fluorurada de acuerdo a lo que informa la literatura $(18 \% \text { y } 60 \%)^{2}$. La condición de empleo del coordinador del programa también fue cambiada (5\% tiempo completo contra 15\% tiempo completo).

\section{RESULTADOS}

En la Tabla 2 se presenta un resumen de la estimación de costo para operar un programa de fluoruración del agua de beber por seis años para 80.000 niños. Se estimó un valor para un programa de seis años de RCH\$ 67.306.224. Esto equivale a aproximadamente RCH\$140,22 por año por niño.

Los costos del tratamiento dental se calcularon usando los costos locales reales para obturaciones y extracciones. Se tomó en cuenta los costos para la familia por tratamiento, en términos de pérdida sueldo/producción, como también los costos de transporte para viajar al lugar de tratamiento dental. La Tabla 3 muestra el costo estimado de los tratamientos dentales durante los seis años para el grupo bajo el programa y para el grupo status-quo, usando una tasa de descuento del tres por ciento. Los costos fueron $60,9 \%$ más altos en el grupo de comparación (RCH\$ 2.474.207.965 ó RCH\$ $5.154,60$ por niño por año) comparados con el grupo con agua fluorurada (RCH\$ 1.506.608.022 o RCH\$3.138,77 por niño por año).

\section{Cociente costo-efectividad}

Al combinar los costos de operación del programa preventivo con los costos del tratamiento odontológico y al compararlos con los costos del grupo de comparación se obtuvo como resultado un ahorro neto total de RCH\$900.293.719 (o

Tabla 2. Resumen de costos asociados al programa de fluoruración del agua durante seis años

\begin{tabular}{|c|c|c|}
\hline Costos del personal & Cantidad (RCH\$ $*)$ & $\%$ del total \\
\hline Coordinador del Programa ${ }^{a}$ & 3.968 .194 & $5,90 \%$ \\
\hline Dentista examinador $\mathrm{b}$ & 507.806 & $0,75 \%$ \\
\hline Asistente dental $\mathrm{b}$ & 128.472 & $0,20 \%$ \\
\hline Análisis de datos ${ }^{\mathrm{C}}$ & 840.000 & $1,24 \%$ \\
\hline \multicolumn{3}{|l|}{ Servicios de laboratorio } \\
\hline Análisis de orina $\mathrm{d}$ & 1.157 .390 & $1,72 \%$ \\
\hline Análisis de agua $\mathrm{e}$ & 578.695 & $0,86 \%$ \\
\hline \multicolumn{3}{|l|}{ Insumos } \\
\hline Arriendo de oficina mas servicios $f$ & 727.502 & $1,08 \%$ \\
\hline Gastos de oficina $g$ & 330.683 & $0,49 \%$ \\
\hline Instrumental dental $\mathrm{h}$ & 600.000 & $0,89 \%$ \\
\hline Gastos de exámenes dental ${ }^{\mathrm{i}}$ & 55.671 & $0,08 \%$ \\
\hline Insumos del programa flúor $j$ & 58.411 .811 & $86,79 \%$ \\
\hline TOTAL & 67.306 .224 & $100 \%$ \\
\hline
\end{tabular}

* $(\mathrm{RCH} \$)$ [1 US $\$=\mathrm{RCH} \$(2009)$ 600.00]

a Salario del coordinador está basado en el salario de un dentista trabajando en el sector público

b Salario del Dentista y assitente dental están basado en los salarios del sector público.

c Costo estándar cobrado por el Instituto de el Instituto de Nutrición y Tecnología de los Alimentos, Universidad de Chile.

d Vigilancia epidemiológica en basa lineamentos del Ministerio de Salud para comunidades bajo programas de fluoruración. Costo estándar cobrado por el Instituto de Nutrición y Tecnología de los Alimentos, Universidad de Chile: RCH\$5.284 por análisis de muestra de orina.

e Normas estándares para muestreo de uso en Chile indican que 20 muestras al año es aceptable (Instituto Nacional de Normalización, 1994). Costo estándar cobrado por el Instituto de Nutrición y Tecnología de los Alimentos, Universidad de Chile RCH\$ 7,350.

f Alquiler de oficina se calculó usando tasas comerciarles estándares, bajo un uso del 10\% del tiempo.

$\mathrm{g}$ Incluye el mobiliario de oficina y los costos generales, como electricidad, etc. calculados usando costos estándares, bajo un uso del $10 \%$ del tiempo.

h ncluye 100 espejos y sondas dentales.

i Incluye guantes, máscaras, toallas de papel, y otros materiales y equipamientos desechables.

j Costo por metro cúbico de agua por adicionar fluoruros por consumo de agua en niños de 6-12 de edad. 
Tabla 3. Resumen de los costos de tratamiento dental en las comunidades estudio y control después de seis años de programa

\begin{tabular}{|l|r|r|}
\hline Tratamiento & Agua fluorurada $(\mathrm{RCH} \$)^{\mathrm{d}}$ & ${\text { Satus-quo }(\mathrm{RCH} \$)^{\mathrm{d}}}^{\mathrm{a}}$ \\
\hline Restauraciones $^{\mathrm{a}}$ & 1.018 .503 .050 & 1.623 .946 .530 \\
\hline Extracciones $^{\mathrm{a}}$ & 16.306 .337 & 60.566 .393 \\
\hline Traslados $^{\mathrm{b}}$ & 288.575 .864 & 483.017 .356 \\
\hline Pérdidas laborales $^{\mathrm{c}}$ & 183.222 .771 & 306.677 .686 \\
\hline Total costos & 1.506 .608 .022 & 2.474 .207 .965 \\
\hline
\end{tabular}

a Costo de tratamientos en base a aranceles del sector público. RCH\$ 7,700 por obturación. Exodoncias se basan en el costo of extracción simple. RCH\$ 3,170 por extracción. Ambos procedimientos incluyen los costos del examen dental.

b Costo estimado para viaje de padre y niño: RCH $\$ 2,100$ por viaje.

c Salario mínimo mensual en 2009 (RCH\$160,000). Costo del tiempo de viaje y tratamiento RCH\$952 por hora.

d Costo estimado calculado multiplicando el componente incremento promedio del COPD por costos de tratamiento dental.

RCH\$ 11.254,00 por niño; RCH\$2.474.207.965 - RCH\$ 1.574.014.244/80.000) atribuible al programa preventivo luego de seis años. De esta forma, una inversión pública de RCH\$ 140,22 (67.306.224/80.000/6) por año por niño, tuvo como resultado una reducción en los costos de tratamiento dental por niño por año de RCH\$ 1875,67 [(RCH\$ 2.474.207.965 - RCH\$ 1.574.014.244)/80.000/4].

La Tabla 4 (línea E) resume el cociente incremental costo-efectividad para el grupo de intervención comparándolo con el grupo de comparación para todo el programa. La tasa total estimó un ahorro de RCH\$ 8.930,49 por COPD [ $\mathrm{RCH} \$ 2.474 .207 .965$ - RCH\$1.574.014.244)/100.800]. De forma tal que, una inversión de RCH\$140,22 anual por niño obtuvo un ahorro neto de RCH\$ 8.930,49 por COPD evitado. Por lo tanto, el programa de fluoruración del agua de beber representa, no sólo una reducción en la enfermedad, sino que también un ahorro neto para la comunidad.

El análisis de sensibilidad indica que la variación de las suposiciones, tanto en el lado de los costos, como en el lado de los resultados de la ecuación, resulta en un cociente incremental costo-efectividad que varió entre RCH\$ 7.950,30 y RCH\$ 10.121,33 ahorrados por COPD evitado. El resultado menos favorable se estableció usando el límite inferior del supuesto de efectividad (18\%) y una tasa de descuento del $0 \%$. En forma inversa,

Tabla 4. Costos totales, beneficios totales y cociente de costo-efectividad para la totalidad del programa de fluorización del agua $\mathrm{RCH} \$$

\begin{tabular}{|c|l|r|}
\hline A & Costos totales comunidad intervenida & 1.574 .014 .244 \\
\hline B & Costos totales comunidad control & 2.474 .207 .965 \\
\hline C & Costos netos (o ahorros) & $(900.293 .719)$ \\
\hline & Ahorro por niño luego de 6 años & 11.254 .00 \\
\hline & Costo del programa por niño & 841,33 \\
\hline & Costo del programa por niño por año & 140,22 \\
\hline D & Beneficios incrementales (COPD evitados: 1,26 x 80.000) & 100.800 \\
\hline E & Costos incrementales (o ahorros) RCH\$ por COPD evitado & $(8.930,49)$ \\
\hline
\end{tabular}

A. El costo de la comunidad bajo el programa es la suma de los costos del programa, Tabla 2, más el costo de los tratamientos Tabla 3.

B. El costo de la comunidad de comparación es el costo de los tratamientos Tabla 3.

D. El beneficio incremental se calculo multiplicando la diferencia anual en el COPD entre la comunidad bajo el programa y la comunidad de comparación (de la Tabla 1) por 80.000 . 
el resultado más favorable se obtuvo al usar el extremo superior de efectividad en la reducción de caries $(60 \%)$ y una tasa de descuento del $6 \%$.

\section{DISCUSIÓN}

Nuestro análisis primario estimó que si un programa de prevención de caries que utiliza agua como el vehículo para el fluoruro a la comunidad estuviera disponible para 80.000 niños desde los 6 a los 12 años de edad, el ahorro neto, desde una perspectiva societal, sería de RCH\$ 8.930,49 por COPD evitado luego de seis años, comparado a un grupo similar no expuesto al programa (o statu quo). Estos ahorros para la sociedad se logran a un costo anual de RCH\$140,22 por niño. Como promedio, este programa produciría un retorno para la sociedad en costos de tratamiento dentales equivalente a RCH\$1.875,67 por niño por año.

El cociente costo-efectividad se mantuvo positivo sobre la alternativa, esto es ahorros, incluso cuando se usaron supuestos más pesimistas que aquellos para un "caso típico".

Sin embargo, este cociente se puede considerar como un cálculo conservador. Por ejemplo, sólo se consideró el costo del tratamiento inicial. Sin embargo, no es inusual que una restauración deba ser reemplazada. Costos adicionales debido a tratamientos más costosos tampoco fueron considerados (por ejemplo, el costo de tratamientos de endodoncia, incrustaciones, etc.). Además, todas las restauraciones fueron consideradas como obturaciones de una superficie. Esto se debió a que bajo el arancel del servicio público chileno, el costo de una restauración es independiente del número de superficies de diente o la complejidad de tratamiento. Por tanto, es muy probable que esto haya resultado en una subvaloración del cociente y del costo evitado en tratamientos dentales.

Este estudio representa un paso inicial para determinar si un programa preventivo comunitario, como el agua fluorurada, representa un uso eficiente de los recursos de la sociedad. Para la mayoría de supuestos del modelo hubo datos disponibles, sin embargo, se requieren estudios longitudinales para definir algunas características con más precisión. Así, estudios se deben realizar para reducir la del uso de supuestos?.

Este estudio es una de las pocas evaluaciones económicas de programa de prevención de salud bucodental en Chile, lo cual destaca la necesidad de estudios que evalúen sistemáticamente los costos y las consecuencias de programas alternativos para mejorar la salud bucodental de la comunidad que reflejen la situación y las prácticas chilenas habituales.

Por otra parte, aunque la caries dental continúa siendo un problema serio de salud pública a nivel mundial, la situación y las necesidades de salud no son constantes en el tiempo. No obstante, hasta la fecha han habido pocos estudios centrados en cómo la disminución de la caries dental, conjuntamente con una mayor retención de diente naturales ha afectado la economía de la fluoración del agua en una población que envejece ${ }^{12}$.

En conclusión, aunque el análisis tiene limitaciones inherentes como consecuencia de su dependencia en un número de supuestos, los hallazgos indican que el programa de fluoruración del agua de beber es una medida costo-efectivo, que conduce a un mejoramiento de la salud dental de la población, y a una reducción de los costos totales (es decir, ahorros) para la sociedad.

Agradecimiento: Parte del material que se presenta fue publicado como parte de un estudio comparativo de diferentes modalidades de prevención de caries. Agracemos a la revista Community Dental Health por permitirnos su uso en esta publicación.

\section{REFERENCIAS}

1. World Health Organization Fluorides and Oral Health. Report of a WHO expert committee on oral health status and fluoride use. (Technical Report Series 846). Geneva: WHO; 1994.

2. Centers for Disease Control and Prevention. Recommendations for using fluoride to prevent and control dental caries in the United States. MMWR. 2001; 50(RR14):1-42.

3. Centers for Disease Control and Prevention. Fluoridation of public drinking water to prevent dental caries. MMWR. 1999;48:933-90.

4. Ministerio de Salud de Chile. Normas de uso de fluoruros en la prevención odontológica. Santiago: Departamento Odontológico, Ministerio de Salud de Chile; 1998.

5. Ministerio de Salud de Chile. Objetivos sanitarios y modelos de salud para la década 2000-2010. Santiago: Ministerio de Salud de Chile; 2002. 
6. Barros L, Jiménez J, Risnik A. Report on dental caries and other oral diseases in schoolchildren from Curico and San Fernando [In Spanish]. Revista de Medicina Preventiva y Social del SNS. 1964:4:3-4.

7. Urbina T, Vincent M, Nacrur JP, Vargas S. Dental caries in pre-school and basic school children from Santiago [In Spanish]. Rev Dental Chile. 1987;18:43-58.

8 Gómez S, Fernández O. Fluoración del agua potable experiencia en Chile. En: Gómez S, ed. Fluorterapia en odontología para niños y adultos. 4a ed.; 2010. Pp. 211-21.

9. Drummond M, Sculpher M, Torrance G, O'Brien B, Stoddart G. Methods for the economic evaluation of health care programmes, $3 \mathrm{a}$ ed. Oxford: Oxford University Press; 2005.

10. Niessen LC, Douglass C. Theoretical considerations in applying benefit-cost and cost-effectiveness analyses to preventive dental programs. J Public Health Dent 1984;44:156-68.

11. Griffin SO, Jones K, Tomar SL. An economic evaluation of community water fluoridation. J Public Health Dent. 2001;61:78-84.

12. Campain A, Mariño R, Wright FAC, Harrison D, Bailey D, Morgan M. The impact of changing dental needs on cost savings from fluoridation. Aust Dent J. 2010;55:37-44.

13. Báez R. Análisis institucional para el desarrollo de un programa nacional de fluoruración de la sal en Honduras. Washington, D.C.: OPS; 1996.

14. Sosa M, García M, Gómez A, Cuéllar L, Mojáiber A. Factibilidad del programa de fluoruración de la sal de consumo humano en Cuba. Rev Cub Salud Publica. 2004;30. Disponible en: http:// bvs.sld.cu/revistas/spu/vol30_1_04/sp08104. htm

15. Mariño R, Fajardo J, Arana A, Garcia C, Pachas F. Economic evaluation of a salt fluoridation program in Peru. J Public Health Dent. 2011;71:125-30.

16. Mariño R, Fajardo J, Morgan M. Economic evaluation of dental caries prevention programs using milk and its products as the vehicle for Fluorides: cost versus benefits. En: Watson R, Gerald J, Preedy, V eds. Dietary Supplements, and Nutriceuticals: Cost Analysis versus Clinical Benefits (editorial). Springer: Humana Press. 2011.p.143-61.

17. Mauro S, García E, Cinque C, Squassi A, Bordoni N. Eficiencia de tres fluoruros concentrados para la estabilización de caries de esmalte. Bol Asoc Argent Odontol Ninos. 2004;33:4-11.

18. Murray C, Evans DB, Acharya A, Baltussen R. Development of WHO guidelines on generalized cost-effectiveness analysis. Health Econ. 2000;9:235-51.

19. Superintendencia de Servicios Sanitarios. Informe de Gestión del sector Sanitario 2006. Santiago: Superintendencia de Servicios Sanitarios; 2007.

20. Chile, Ministerio De Economía, Fomento Y Reconstrucción. Decreto 169 - Fija formulas tarifarias de los servicios de producción y distribución de agua potable y recolección de aguas servidas (Revisado 2006). Disponible en: http:/ / www.siss.gob.cl/transparencia/terceros/decretos_tar/Maip $\%$ C3\%BA\%20-\%20SMAPA-169. pdf.

21. Ministerio de Salud de Chile. Estudios de excreción urinaria de flúor en niños pre-escolares. (Parte 2). Santiago: Departamento Odontológico, Ministerio de Salud de Chile; 1998.

22. Instituto Nacional de Normalización. Selección de muestras al azar (NCH 43.Of61). $2^{\mathrm{a}}$ ed. Santiago: Instituto Nacional de Normalización; 1994.

23. Urbina T, Caro JC, Vincent M. Caries Dentaria y Fluorosis en niños de 6 a 8 y 12 años, de la II, VI, VIII, IX, X, y Región Metropolitana. Chile. Santiago: Departamento Odontológico, Ministerio de Salud de Chile; 1997.

24. Soto LQ, Tapia RC, Jara GB, Rodríguez GP, Urbina TR. Diagnóstico nacional de salud bucal del adolescente de 12 años y evaluación del grado de cumplimiento de los objetivos sanitarios de salud bucal 2000 - 2010 [serie de documentos tecnicos]. Santiago: Universidad Mayor: Facultad de Odontología; 2007.

25. Drummond M, \& Jefferson T. Guidelines for authors and peer reviewers of economic submissions to BMJ. BMJ. 1996;313: 275-84. 